\title{
MODELS OF LOW REYNOLDS NUMBER SWIMMERS INSPIRED BY CELL BLEBBING
}

\author{
QIXUAN WANG, JIFENG HU AND HANS OTHMER \\ SCHOOL OF MATHEMATICS, UNIVERSITY OF MINNESOTA, USA
}

\begin{abstract}
Eukaryotic cells move through the complex micro-environment of a tissue either by attaching to the extracellular matrix - sometimes degrading it locally - and pulling themselves along, or by squeezing through the matrix by appropriate sequences of shape changes. Some cells can even swim by shape changes, and one mode used is called blebbing, in which a cell creates a small hemispherical protrusion that may grow to incorporate the entire cell volume or may be reabsorbed into the primary volume. Herein we develop and analyze several models for swimming at low Reynolds number inspired by cell blebbing. These models comprise several connected spheres, and each connected pair of spheres can exchange volume with their complement in the pair. We show that the cell can propel itself through the fluid using a suitable sequence of volume exchanges, and we evaluate the efficiency of this mode of swimming.
\end{abstract}

Key words. cell protrusion, micro-swimmer, Stokes solution, linked-sphere models

AMS(MOS) subject classifications. Primary 76Z10, 49J20, 92C17, 93B05.

1. Introduction. Cell locomotion is essential for embryonic development, angiogenesis, tissue regeneration, the immune response, and wound healing in multicellular organisms, and plays a very deleterious role in cancer metastasis in humans. Locomotion involves the detection and transduction of extracellular chemical and mechanical signals, integration of the signals into an intracellular signal, and the spatio-temporal control of the intracellular biochemical and mechanical responses that lead to force generation, morphological changes and directed movement [10]. While many single-celled organisms use flagella or cilia to swim, there are two basic modes of movement used by eukaryotic cells that lack such structures mesenchymal and amoeboid [4]. The former, which can be characterized as 'crawling' in fibroblasts or 'gliding' in keratocytes, involves the extension of finger-like filopodia or pseudopodia and/or broad flat lamellipodia, whose protrusion is driven by actin polymerization at the leading edge. This mode relies on strong adhesion to the substrate, and dominates in cells such as fibroblasts crawling on a $2 \mathrm{D}$ substrate. In the amoeboid mode, which does not rely on strong adhesion, cells are more rounded and employ shape changes to move - in effect 'jostling through the crowd' or 'swimming'. Recent experiments have shown that numerous cell types display enormous plasticity in locomotion, in that they sense the mechanical properties of their environment and adjust the balance between the mesenchymal and amoeboid modes accordingly by altering the balance between parallel signal transduction pathways [9]. Pure crawling and pure swimming are the extremes on a continuum of locomotion strategies, but cells can sense their environment and use the most efficient strategy in a given context. 
Some cells produce membrane 'blisters' called blebs, in which the membrane detaches from the cortex locally and the pressure generated by the cortex forces the membrane outward. Fig. 1(a) shows a Dictyostelium discoideum (Dd) cell that uses blebs to extend the leading edge while moving. Blebbing is a specialized form of shape change that may produce movement, but to understand when it does, and to understand movement more generally, one has to integrate the cellular dynamics with the dynamics of the surrounding complex medium, the ECM. Here we begin with swimming, motivated by recent experiments which show that both neutrophils and Dd can swim - in the strict sense of propelling themselves through a fluid without using any attachments - in response to chemotactic gradients [3]. The experimental observations show a very complex sequence of shape changes in Dd, but we first study how shape changes in abstract models of swimmers can lead to movement in a viscous fluid. Interest in this classical problem stems from the description

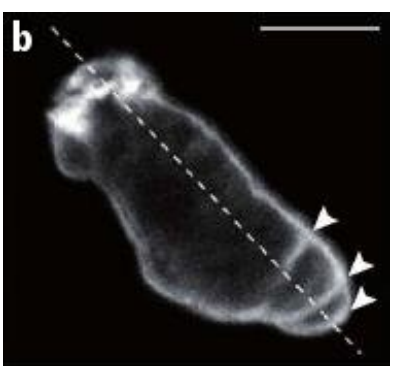

FIG. 1. The actin cortex of a blebbing Dd cell migrating to the lower right. White arrowheads indicate the successive blebs and arcs of the actin cortex (from [12]). of life at low Reynolds number by Purcell [8].

The essential ideas are as follows - the current state of knowledge is reviewed elsewhere [7].

The governing equations for an incompressible Newtonian fluid of density $\rho$ and viscosity $\mu$ are given by

$$
\rho \frac{\partial \mathbf{v}}{\partial t}+\rho(\mathbf{v} \cdot \nabla) \mathbf{v}=-\nabla p+\mu \Delta \mathbf{v}+\mathbf{f}, \quad \nabla \cdot \mathbf{v}=0
$$

where $\mathbf{f}$ is the external force field. The Reynolds number based on a characteristic length scale $L$ and speed scale $V$ is $\operatorname{Re}=\rho \mathrm{LV} / \mu$, and nondimensionalization of Eq. (1.1) shows that when Re $\ll 1$ the acceleration terms can be ignored. This defines a low Reynolds number (LRN) flow. When there are no external force fields, as we will assume hereafter, the equations simplify to the Stokes equations

$$
\mu \Delta \mathbf{v}-\nabla p=\mathbf{0}, \quad \nabla \cdot \mathbf{v}=0 .
$$

The small size and slow speed of cells considered here leads to LRN flows, and in this regime cells move by exploiting the viscous resistance of the fluid. However, since time is absent from the equations, a time-reversible stroke produces no net motion, which is the content of the famous 'scallop theorem' [8]. Because there is no net force or torque on a swimmer in the Stokes regime, movement is a purely geometric process: the net displacement of a swimmer during a stroke is independent of the rate at which the 
stroke is executed, as long as the Reynolds number remains small enough. The properties of the exterior fluid come into play only when addressing the efficiency of a stroke.

A swimming stroke is defined by a time-dependent sequence of shapes, and a cyclic swimming stroke is a swimming stroke for which the initial and final shapes are identical. Let $\mathcal{B}(t)$ and $\mathbf{V}$ be the boundary and velocity of the swimmer, respectively. $\mathbf{V}$ can be decomposed into a part $\mathbf{v}$ that corresponds to the intrinsic shape deformations, and a part $\mathbf{U}$ that corresponds to a rigid motion. Then most LRN self-propulsion problems can be stated as: given a cyclic shape deformation by specifying $\mathbf{v}$, solve the Stokes equations subject to

$$
\sum \mathbf{F}_{i}=0, \quad \sum \boldsymbol{\Gamma}_{i}=0,\left.\quad \mathbf{u}\right|_{\mathcal{B}}=\mathbf{V},\left.\quad \mathbf{u}\right|_{\mathbf{x} \rightarrow \infty}=\mathbf{0}
$$

where $\mathbf{u}$ is the velocity field of the surrounding fluid, and $\mathbf{F}_{i}$ and $\boldsymbol{\Gamma}_{i}$ are forces and torques on the swimmer.

Purcell's model swimmer comprises three connected, rigid links constrained to move in a plane, where adjacent links do a constrained rotation around joints and can thereby change the angle between them. Here the shape is specified by two parameters, the angles between adjacent links, and Purcell showed that one can impose sequences of changes in the angles that produce motion of the swimmer. Despite its geometric simplicity, the relationships between geometric parameters, speed and efficiency of swimming are not simple, and various simpler models such as the three-linked-spheres model and the push-me-pull-you model have appeared since [5].

As an example, consider a swimmer made of three spheres and two rods connecting them, all immersed in a LRN fluid (Fig. 2). Assume that the lengths $L_{1}$ and

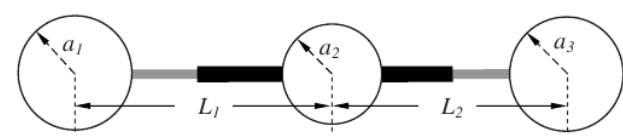

FIG. 2. The three-linked-spheres swimmer[6]. $L_{2}$ of the rods connecting them are changed in a prescribed way. Then for a large separation one can use the Oseen tensor, which defines the leading-order term for $\mathbf{u}$, to relate the forces $f_{i}$ on the spheres to the speed $v_{1}$ of the first sphere as follows $[6]$ :

$$
v_{1}=\frac{f_{1}}{6 \pi \mu a_{1}}+\frac{f_{2}}{6 \pi \mu L_{1}}+\frac{f_{3}}{6 \pi \mu\left(L_{1}+L_{2}\right)} .
$$

There are similar equations for $v_{2}$ and $v_{3}$, and the leading order approximation $V_{0}$ to the speed $V$ is the mean speed $V_{0}=\sum_{i} v_{i} / 3$. After specifying the velocities $L_{i}^{\prime}$ and using the force-free condition at Eq. (1.3), one can eliminate the forces, and when all spheres have the same radius $a$ one finds that

$$
V_{0}=\frac{a}{6}\left[\left(\frac{L_{2}^{\prime}-L_{1}^{\prime}}{L_{1}+L_{2}}\right)+2\left(\frac{L_{1}^{\prime}}{L_{2}}-\frac{L_{2}^{\prime}}{L_{1}}\right)\right]
$$


plus terms that average to zero over a cycle [6]. Thus the swimmer can move for suitable choices of the velocities $L_{i}^{\prime}$. The efficiency of swimming for this model has been estimated, and an algorithm for finding optimal strokes exists [1].

2. The linear $n$-sphere swimmer with volume exchange. As a generalization of the previous example that is more directly related to cell motility and blebbing, we consider a connected linear chain of spheres, each of which can exchange mass with its neighbors. We assume that the distance between their centers of mass remains unchanged, and that the distance between each pair is much larger than the radii of the spheres. It follows from the scallop theorem that a two-sphere model cannot swim, since it has only one degree of freedom, and therefore a minimal model must comprise at least three spheres, as in the previous example.

Here we restrict attention to the 3-sphere swimmer shown in Fig. 3, immersed in a Newtonian fluid with no-slip boundary conditions at the boundary of the spheres. The connecting rods are massless, the distance

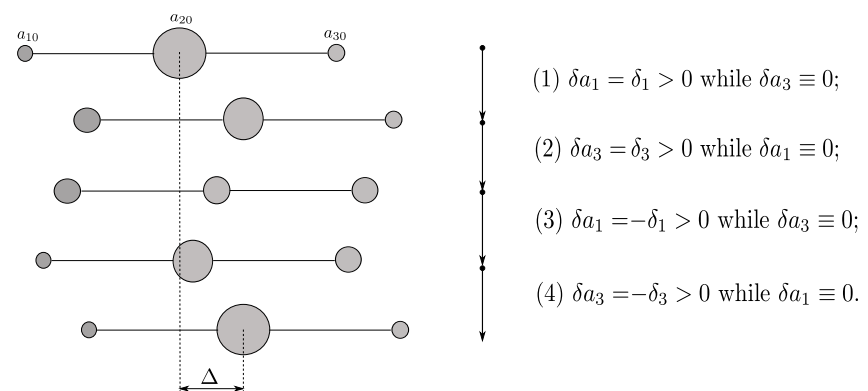

FIG. 3. A cycle of the swimmer

$l$ between the centers of two adjacent spheres is constant, and for simplicity we assume that during the volume exchange process between adjacent spheres, the volumes remain spherical. Let $a_{i}$ be the radius of the $i$ th sphere and assume that $\varepsilon \sim a_{i} / l \ll 1$.

The solution to Eq. (1.2) for the flow around a single sphere of radius $a$ subject to a force $\mathbf{f}$ and dilated at the rate $\dot{v}$ is

$$
\mathbf{u}(\mathbf{r} ; a, \mathbf{f}, \dot{v})=\frac{1}{24 \pi \mu}\left[\left(3+\xi^{2}\right) \frac{\mathbf{f}}{r}+3\left(1-\xi^{2}\right) \frac{(\mathbf{f} \cdot \mathbf{r}) \mathbf{r}}{r^{2}}\right]+\frac{\dot{v}}{4 \pi r^{2}} \mathbf{r}
$$

where $\mathbf{u}(\mathbf{r} ; a, \mathbf{f}, \dot{v})$ is the velocity at position $\mathbf{r}$ from the center of the sphere $[2]$ and $\xi \equiv a / r$.

In the linear case, by symmetry, both the net velocities of the spheres and the net forces acting on each sphere should be parallel to the symmetry axis, thus can be taken as scalars. Let $f_{i}$ be the net force acting on the $i$ th sphere by the surrounding Stokes fluid. It is reasonable to assume that 
$O\left(f_{1}\right) \sim O\left(f_{2}\right) \sim O\left(f_{3}\right)$. To leading order in $\varepsilon \sim a_{i} / l$,

$$
\left\{\begin{aligned}
U_{1} & \sim \frac{f_{1}}{6 \pi \mu a_{1}}-\frac{\dot{v}_{2}}{4 l^{2}}-\frac{\dot{v}_{3}}{16 l^{2}} \\
U_{2} & \sim \frac{f_{2}}{6 \pi \mu a_{2}}+\frac{\dot{v}_{1}}{4 l^{2}}-\frac{\dot{v}_{3}}{4 l^{2}} \\
U_{3} & \sim \frac{f_{3}}{6 \pi \mu a_{3}}+\frac{\dot{v}_{1}}{16 l^{2}}+\frac{\dot{v}_{2}}{4 l^{2}}
\end{aligned}\right.
$$

where $U_{i}$ is the velocity at the center of the $i^{\text {th }}$ sphere.

The swimming velocity of the whole object is the mean translational velocity, i.e., $\bar{U}=\left(U_{1}+U_{2}+U_{3}\right) / 3$ Because we assume that the length of the two connecting arms is always $l$, we have $i=U_{2}-U_{1}=U_{3}-U_{2}=0$, and therefore

$$
U_{1}=U_{2}=U_{3}=\bar{U}
$$

Since the system is force-free $f_{1}+f_{2}+f_{3}=0$ and because the total volume $V$ is conserved

$$
\dot{v}_{1}+\dot{v}_{2}+\dot{v}_{3}=0 \quad \text { or } \quad a_{1}^{2} \dot{a}_{1}+a_{2}^{2} \dot{a}_{2}+a_{3}^{2} \dot{a}_{3}=0
$$

From the foregoing we find that

$$
\bar{U}=\frac{\left(a_{1}+a_{2}-\frac{3}{4} a_{3}\right) \dot{v}_{1}-\left(a_{3}+a_{2}-\frac{3}{4} a_{1}\right) \dot{v}_{3}}{4 l^{2}\left(a_{1}+a_{2}+a_{3}\right)}
$$

where $a_{2}=\left(3 V /(4 \pi)-a_{1}^{3}-a_{3}^{3}\right)^{1 / 3}$

To propel the swimmer with $\dot{v}_{i}$ given, the power required is

$$
P=\frac{\mu}{\pi}\left[\left(\frac{1}{a_{1}^{3}}+\frac{1}{a_{2}^{3}}\right) \dot{v}_{1}^{2}+\frac{2}{a_{2}^{3}} \dot{v}_{1} \dot{v}_{3}+\left(\frac{1}{a_{2}^{3}}+\frac{1}{a_{3}^{3}}\right) \dot{v}_{3}^{2}\right]
$$

and the efficiency of a stroke $\gamma$ is [2]

$$
e(\gamma):=\frac{6 \pi \mu X^{2}(\gamma)}{\tau \int_{0}^{\tau} P d t}
$$

Some analytical results on movement can be obtained - detailed proofs of the folllowing will appear elsewhere [11]. Let $\bar{d} X>0$ represent an infinitesimal displacement to the right in Fig. 3. Given an infinitesimal shape change $\left(d a_{1}, d a_{3}\right)$, it follows from Eq. (2.5) that

$$
\bar{d} X=\frac{\pi}{l^{2}}\left[a_{1}^{2}\left(1-\frac{7}{4} \frac{a_{3}}{a_{1}+a_{2}+a_{3}}\right) d a_{1}-a_{3}^{2}\left(1-\frac{7}{4} \frac{a_{1}}{a_{1}+a_{2}+a_{3}}\right) d a_{3}\right]
$$

Using Stokes' theorem, the translation $\delta X$ associated with a closed loop is

$$
\delta X=\frac{7 \pi}{4 l^{2}}\left[a_{1}^{2} \partial_{a_{3}} \frac{a_{3}}{a_{1}+a_{2}+a_{3}}+a_{3}^{2} \partial_{a_{1}} \frac{a_{1}}{a_{1}+a_{2}+a_{3}}\right] d a_{1} \wedge d a_{3},
$$

where $d a_{1} \wedge d a_{3}$ denotes the signed area enclosed by the loop. Some conclusions that can be drawn from this are as follows. 
- When only one $d a_{i}$ is non-zero, the direction of movement is always from the expanding sphere to the contracting one, provided that the center sphere is large enough. An intuitive explanation of this is [2]: the expanding sphere acts as a source pushing away the shrinking sphere which acts as a sink to pull the expanding sphere.

- For any stroke $\gamma$ homotopic to $S^{1}$ in the $\left(a_{1}, a_{2}\right)$ plane, an increase of the stroke amplitude will increase the net translation per stroke, while an increase in the initial radius $a_{20}$ of the central sphere (with $a_{10}$ and $a_{30}$ fixed) will decrease the net translation per stroke. A firstorder approximation to the displacement is $|X(\gamma)| \sim \frac{\varepsilon}{l} \operatorname{Area}(\Omega)$, where $\varepsilon \sim a_{i} / l, \Omega$ is the region enclosed by $\gamma$, and $\operatorname{Area}(\Omega)$ is the signed area of $\Omega$.

- Increasing the initial radius $a_{20}$ of the central sphere (with $a_{10}$ and $a_{30}$ fixed) decreases the efficiency. Also, for infinitesimal strokes, increasing the stroke amplitude $\left|d a_{1} \wedge d a_{3}\right|$ symmetrically by a factor $r$ (i.e., $\widetilde{d a_{1}}=$ $r d a_{1}$ and $\widetilde{d a_{3}}=r d a_{3}$ ) will increase the efficiency by $r^{2}$. In particular, if we assume that $a_{i} \sim a$ and $d a_{i} \sim d a$, then we have the approximation: $\bar{d} e \sim a|d a|^{2} / l^{4}$.

For finite-amplitude changes we must compute the displacement and efficiency numerically, and for this we consider the cycle shown in Fig. 3 . We suppose that initially $a_{10}=a, a_{20}=s_{2} a$, and $a_{30}=s_{3} a$, where $s_{i}$ measures the initial relative size. At the end of each step within a stroke, the radius of sphere $i(i=1,3)$ is either $a_{i 0}$ or $a_{i 0}+\delta_{i}$. Let $\delta_{1}=r_{1} a, \delta_{3}=r_{3} a$, where $r_{i}$ measures the stroke amplitude, and let $\Delta$ be the net translation after one full cycle. Figs. 4 and 5 show the effect of varying the initial size of the central sphere and the stroke amplitude on the displacement and efficiency.
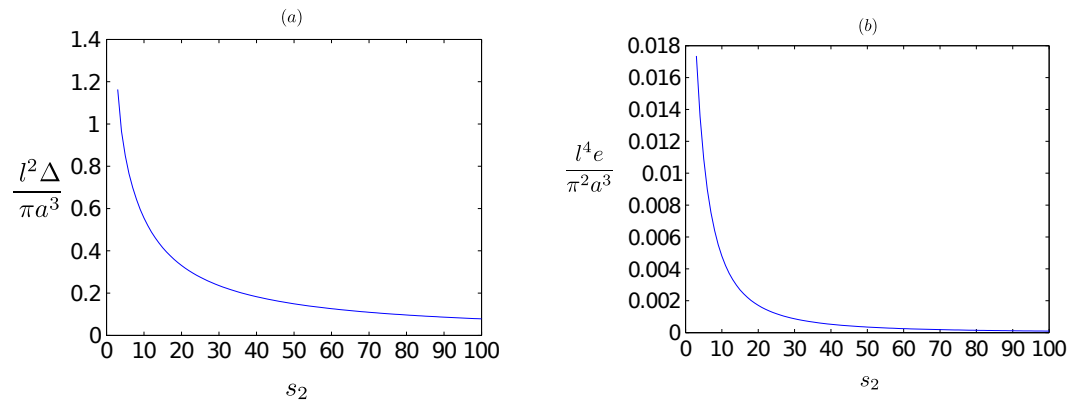

FIG. 4. (a) The relationship between the net translation $l^{2} \Delta /\left(\pi a^{3}\right)$ and the initial size $s_{2}$ of the center sphere, and (b) between the efficiency $l^{4} e /\left(\pi^{2} a^{3}\right)$ and $s_{2}$, for $s_{3}=r_{1}=r_{3}=1$.

Fig. 4 shows that increasing the initial volume of the center sphere will decrease both the net translation and the efficiency of the cycle, whereas in Fig. 5 one sees that increasing the amplitude in each step of the cycle will increase both the net translation of the swimmer after a full cycle and 

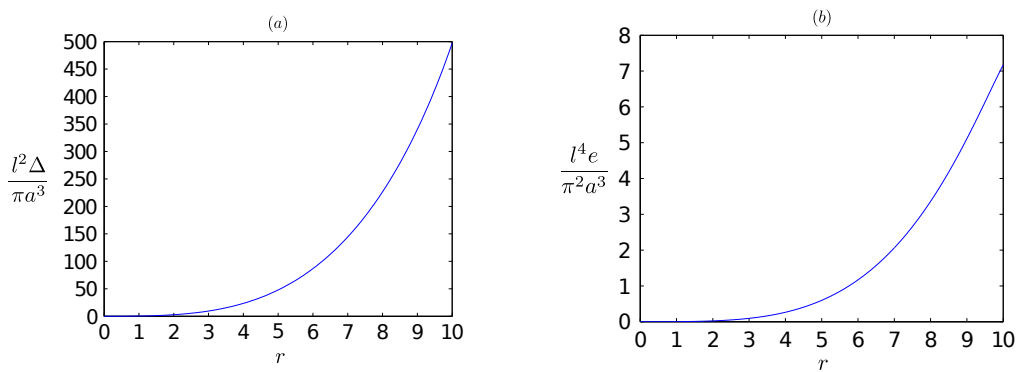

FIG. 5. (a) The relationship between the net translation $l^{2} \Delta /\left(\pi a^{3}\right)$ and the stroke amplitude $r$, and (b) between the efficiency $l^{4} e /\left(\pi^{2} a^{3}\right)$ and $r$, for $s_{3}=1, r_{2}=r_{3}=r$, and $s_{2}=15$.

its efficiency, as predicted by the analysis.

3. The planar 3 -sphere swimmer. Next consider a 3 -sphere swimmer that can move in the plane as shown in Fig. 6, wherein the 1st and 2nd spheres can exchange volume with the 0 th sphere while preserving the total volume. We assume that $a_{i} / l \alpha \ll 1$, and suppose that other constraints and the notation are as before.
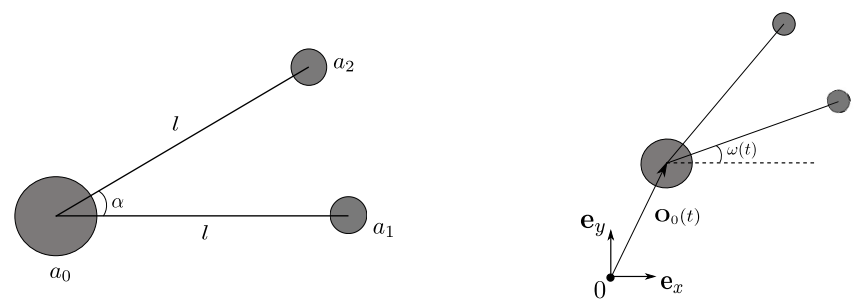

FIG. 6. The planar 3-sphere swimmer.

The motion of the structure can be uniquely determined by $\left(\mathbf{O}_{0} ; \omega\right)$, where $\mathbf{O}_{0}(t)$ is the position of the center of sphere 0 in a global chart, and $\omega(t)$ measures the rotation of the structure with respect to sphere 0 .

If, as before, we solve the Stokes equations plus the volume conservation constraint Eq. (2.4), we obtain

$$
n \dot{\omega}=\frac{a_{0}\left(a_{2} \dot{v}_{1}-a_{1} \dot{v}_{2}\right) \sin \alpha}{4 \pi l^{3}\left[a_{0}\left(a_{1}+a_{2}\right)+2 a_{1} a_{2}(1-\cos \alpha)\right]}\left(\frac{1}{8 \sin ^{3} \frac{\alpha}{2}}-1\right) .
$$

The assumption that $a_{i} / l \alpha \ll 1$ ensures that $\left(8 \sin ^{3} \frac{\alpha}{2}\right)^{-1}-1$ will not be 
too large, and it follows that

$$
\begin{aligned}
\mathbf{U}_{0}= & -\left(\frac{\dot{v}_{1}}{4 \pi l^{2}}\left[a_{0}+a_{1}+a_{2}\left(\cos \alpha+\frac{1}{4 \sin \frac{\alpha}{2}}\right)\right]+\frac{\dot{v}_{2}}{4 \pi l^{2}}\left[a_{0} \cos \alpha\right.\right. \\
& \left.\left.+a_{1}\left(1-\frac{1}{4 \sin \frac{\alpha}{2}}\right)+a_{2} \cos \alpha\right]-a_{2} l \dot{\omega} \sin \alpha\right)\left(\frac{\cos \omega \mathbf{e}_{x}+\sin \omega \mathbf{e}_{y}}{a_{0}+a_{1}+a_{2}}\right) \\
& -\left(\frac{a_{2} \dot{v}_{1}}{4 \pi l^{2}} \sin \alpha\left(1-\frac{1}{8 \sin ^{3} \frac{\alpha}{2}}\right)+\frac{\dot{v}_{2}}{4 \pi l^{2}}\left(a_{0} \sin \alpha+a_{2} \sin \alpha\right.\right. \\
& \left.\left.+\frac{a_{1}}{4 \sin \frac{\alpha}{2} \tan \frac{\alpha}{2}}\right)+\left(a_{1}+a_{2} \cos \alpha\right) l \dot{\omega}\right)\left(\frac{-\sin \omega \mathbf{e}_{x}+\cos \omega \mathbf{e}_{y}}{a_{0}+a_{1}+a_{2}}\right)
\end{aligned}
$$

where $\omega(t)=\int_{0}^{t} \dot{\omega} d t$.

As was true for the linear array, some analytical results can be obtained [11]. From Eq. (3.1), we have

$$
\bar{d} \omega=\frac{\sin \alpha}{l^{3}}\left(\frac{1}{8 \sin ^{3} \frac{\alpha}{2}}-1\right) \frac{a_{0} a_{1} a_{2}\left(a_{1} d a_{1}-a_{2} d a_{2}\right)}{a_{0}\left(a_{1}+a_{2}\right)+2 a_{1} a_{2}(1-\cos \alpha)}
$$

where $\bar{d} \omega>0$ represents an infinitesimal rotation counterclockwise. The swimming rotation $\delta \omega$ associated to an infinitesimal closed loop is

$$
\delta \omega=\phi \cdot\left(a_{1} \partial_{a_{2}}+a_{2} \partial_{a_{1}}\right) \psi d a_{2} \wedge d a_{1}
$$

where

$$
\begin{aligned}
\phi(\alpha) & =\frac{\sin \alpha}{l^{3}}\left(\frac{1}{8 \sin ^{3} \frac{\alpha}{2}}-1\right) \\
\psi\left(a_{1}, a_{2} ; \alpha\right) & =\frac{a_{0} a_{1} a_{2}}{a_{0}\left(a_{1}+a_{2}\right)+2 a_{1} a_{2}(1-\cos \alpha)} .
\end{aligned}
$$

In Eq. (3.4), $d a_{2} \wedge d a_{1}$ denotes the signed area enclosed by the loop. From this one can conclude that:

(1.) Any linear or equilateral triangular swimmer cannot rotate at any time.

(2.) If the loop is chosen so that $d a_{2} \wedge d a_{1}<0$ and $\mid 2(1-$ $\cos \alpha) \frac{a_{1}^{2} a_{2}^{2}}{a_{0}^{4}} \mid<1$ holds throughout the stroke, then the swimmer rotates clockwise when $\alpha<\pi / 3$, and rotates counterclockwise when $\pi / 3<\alpha<\pi$.

(3.) When $\alpha$ satisfies the standing constraint, we have

$$
\delta \omega \sim \frac{\varepsilon}{l^{2}} \sin \alpha\left(\frac{1}{8 \sin ^{3} \frac{\alpha}{2}}-1\right)\left|d a_{2} \wedge d a_{1}\right|
$$

which implies that either increasing $a_{i}$ or increasing the stroke amplitude $\left|d a_{2} \wedge d a_{1}\right|$ will increase the net rotation $\delta \omega$ of a stroke. 
To extend these results to finite changes, consider a full cycle comprising the following four steps.

(1) sphere 1 transfers volume $\delta_{1}>0$ to sphere 0 ;

(2) sphere 2 transfers volume $\delta_{2}>0$ to sphere 0 ;

(3) sphere 0 transfers volume $\delta_{1}$ back to sphere 1 ;

(4) sphere 0 transfers volume $\delta_{2}$ back to sphere 2 .

We assume that the initial state of a stroke is $\left(\mathbf{O}_{0}, \omega\right)(t=0)=(\mathbf{0}, 0)$, and let $\Omega=\omega(T)$ and $\boldsymbol{\Delta}=\mathbf{O}_{0}(T)$ be the rotation angle and the net translation of the swimmer at the end of the stroke, respectively, where $T$ is the period of the stroke. We express $\boldsymbol{\Delta}$ in global polar coordinates as $\boldsymbol{\Delta}=R \cdot \mathbf{e}_{r}+\Theta \cdot \mathbf{e}_{\theta}$. Fig. 7 illustrates some of the computational results
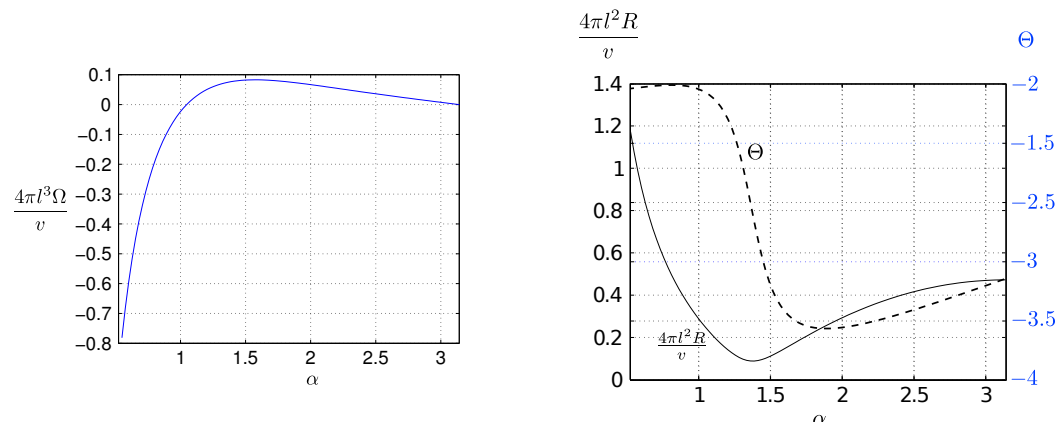

FIG. 7. The rotation $4 \pi l^{3} \Omega(\alpha) / v$ (left) and the translation $4 \pi l^{2} R(\alpha) / v$ and $\Theta(\alpha)$ (right) of the swimmer as a function of the acute angle $\alpha$.

for $\frac{4 \pi l^{3}}{v} \Omega(\alpha), \frac{4 \pi l^{2}}{v} R(\alpha)$ and $\Theta(\alpha)$ with $a_{10}=a_{20}=a, a_{00}=2^{1 / 3} a$, and $\delta_{1}=\delta_{2}=0.9 v$ for $\alpha \in[\pi / 6, \pi]$. From this computational experiment one can conclude the following.

- There are two zeros of $\Omega(\alpha)$, one at $\alpha=\pi / 3$ (corresponding to an equilateral triangle), and the other at $\alpha=\pi$ (a straight line, the case discussed earlier). (This confirms the general result (1) stated above.)

- When $\alpha<\pi / 3, \Omega<0$ (which corresponds to clockwise rotation), and $|\Omega|$ increases rapidly as $\alpha$ decreases. When $\pi / 3<\alpha<\pi, \Omega>0$ (counterclockwise rotation), and $4 \pi l^{3}|\Omega| / v$ has a maximum value of about 0.0830 at $\alpha=\pi / 2$.

- Under the assumption that $\varepsilon \sim a_{i} / l \ll 1, \Omega \sim o\left(\varepsilon^{3}\right)$, which implies that the effect of rotation is not significant.

- $\alpha$ has complicated effects on both the translation distance and the translation direction. $\frac{4 \pi l^{2}}{v} R$ has a minimum of about 0.0884 at around $\alpha=$ $0.44 \pi$; while $\Theta$ has a maximum of about $-0.48 \pi$ at around $\alpha=0.26 \pi$, and a minimum of about $-1.13 \pi$ at around $\alpha=0.60 \pi$.

4. Discussion. In this paper we have briefly sketched some results for a 3-sphere model in which movement results from volume exchange between the spheres. Here we only considered square loops in the control 
space, but an open problem is to determine the optimal loop in the control space. Another generalization under consideration is a model in which volume exchange is coupled with active contraction of the connecting rods to more closely approximate movement in cells. In reality, microorganisms execute much more complicated shape deformations in order to swim, but important insights can be gained from abstract models such as considered here.

Acknowledgments This research was supported in part by NSF grants DMS-0517884 and DMS-0817529.

\section{REFERENCES}

[1] F. Alouges, A. DeSimone, And A. Lefebvre, Optimal strokes for axisymmetric microswimmers, Eur. Phys.l Jour. E: Soft Matter and Biological Physics, 28 (2009), pp. 279-284.

[2] J. E. Avron, O. Kenneth, and D. H. OAknin, Pushmepullyou: an efficient micro-swimmer, New Journal of Physics, 7 (2005), p. 234.

[3] N. P. BARry AND M. S. Bretscher, Dictyostelium amoebae and neutrophils can swim, PNAS, 107 (2010), p. 11376

[4] F. Binamé, G. Pawlak, P. Roux, and U. Hibner, What makes cells move: requirements and obstacles for spontaneous cell motility, Molecular BioSystems, 6 (2010), pp. 648-661.

[5] N. Cohen and J. H. Boyle, Swimming at low Reynolds number: a beginners guide to undulatory locomotion, Contemporary Physics, 51 (2010), pp. 103-123.

[6] R. Golestanian And A. Ajdari, Analytic results for the three-sphere swimmer at low Reynolds number, Phys. Rev. E, 77 (2008), p. 36308.

[7] E. Lauga AND T. R. Powers, The hydrodynamics of swimming microorganisms, Repts. on Prog. in Phys., 72 (2009), p. 096601.

[8] E. Purcell, Life at low reynolds number, Amer.J.Physics, 45 (1977), pp. 3-11.

[9] J. Renkawitz, K. Schumann, M. Weber, T. Lämmermann, H. Pflicke, M. Piel, J. Polleux, J. P. Spatz, And M. Sixt, Adaptive force transmission in amoeboid cell migration, Nature Cell Biology, 11 (2009), pp. 1438-1443.

[10] M. P. Sheetz, D. Felsenfeld, C. G. Galbraith, and D. Choquet, Cell migration as a five-step cycle, in Cell Motility: Control and Mechanism of Motility, J. M. Lackie, G. A. Dunn, and G. E. Jones, eds., Princeton University Press, 1999, pp. 233-243.

[11] Q. WANG, Mathematical and Computational Studies of Cell Motility, PhD thesis, University of Minnesota, 2012.

[12] K. Yoshida And T. Soldati, Dissection of amoeboid movement into two mechanically distinct modes, J. Cell Sci., 119 (2006), pp. 3833-3844. 